\title{
ORIGINAL ARTICLE \\ Arm hand skilled performance in persons with a cervical spinal cord injury-long-term follow-up
}

\author{
AC Franke ${ }^{1}$, GJ Snoek ${ }^{1}$, S de Groot ${ }^{2,3}$, AV Nene ${ }^{1}$, AIF Spooren ${ }^{4}$ and MWM Post ${ }^{5}$
}

Study design: Retrospective cohort study.

Objective: To assess development of arm hand skilled performance (AHSP) during and after in-patient rehabilitation in persons with cervical spinal cord injury (CSCI) and to determine factors that influence the outcome.

Setting: Eight rehabilitation centres in the Netherlands with specialised spinal cord injury departments.

Methods: AHSP was assessed using the Van Lieshout test (VLT) in persons admitted with recent CSCl. Assessment was carried out at the beginning (t1), after 3 months ( $\mathrm{t} 2$ ), at the end ( $\mathrm{t} 3$ ) of in-patient rehabilitation, and 1 and 5 years thereafter ( $\mathrm{t} 4$, $\mathrm{t} 5$ ). Multilevel regression analysis was performed to determine development of AHSP and associations between AHSP and age, gender, motor completeness, lesion level (high or low CSCI), motor scores of upper extremity (MSUE), and pain in the tested arm.

Results: Fifty-five participants were included with mean age 38 years (range 18-64). There were $73 \% \mathrm{male}$, 80\% had high CSCl (C3-C6) and 69\% had motor complete lesion. Scores of VLT improved significantly during in-patient rehabilitation (mean: t1 = 25; $\mathrm{t} 3=33)(P=0.005)$, scores remained unchanged at 1 year $(\mathrm{t} 4=32)$ and 5 years $(\mathrm{t} 5=32)(P=0.903)$ after in-patient rehabilitation. Motor completeness, MSUE and pain were significantly related to the VLT score $(P<0.001, P<0.001, P=0.015$, respectively). Age, gender and lesion level had no significant relationship.

Conclusion: AHSP improved during in-patient rehabilitation. It was then stable during the next 5 years after discharge. Persons with an incomplete lesion, high MSUE and no pain in the tested arm perform best on the VLT.

Spinal Cord (2013) 51, 161-164; doi:10.1038/sc.2012.95; published online 18 September 2012

Keywords: arm hand skilled performance; Van Lieshout test; cervical spinal cord injury; long-term outcome

\section{INTRODUCTION}

The worldwide incidence of spinal cord injury varies between 10.4 and 83 per year per million inhabitants. A significant number of this, almost one-third, is reported to be tetraplegic. ${ }^{1}$ The level of impairment of arm hand function and (in)dependence in self-care relates to the level and completeness of the cervical spinal cord injury (CSCI).,3 Improved arm hand function is positively related to improvement of quality of life. ${ }^{2,4}$ It is also stated that improving arm hand function has the highest priority in persons with chronic CSCI. ${ }^{2,5}$

Only few studies in the medical literature describe long-term outcome of arm hand function in persons with CSCI at activity level. ${ }^{6-10}$ The modified Barthel index and quadriplegia index of function are used as outcome measures in four of these studies. ${ }^{6-9}$ Although they are activity level instruments, they involve more skills than just arm hand function. Harvey et al. ${ }^{10}$ used an activities of daily living test; however, the test was not validated before use. In addition, in the past studies meaning of the term arm hand function was unclear. It did not define whether it was related to the level of body function or activity, according to the International Classification of Functioning, Disability and Health (ICF) model. ${ }^{11}$ To clarify this difference, Spooren et al. ${ }^{12}$ introduced the term 'arm hand skilled performance' (AHSP) which accurately describes the functional possibilities of the arm and hand. The Van Lieshout test (VLT) used in this study, is designed to objectively quantify the quality of movement of the upper extremity at the basic activities level in persons with CSCI. ${ }^{12,13}$ Spooren et al. ${ }^{8}$ measured the AHSP using the VLT. They demonstrated that AHSP improves significantly during in-patient rehabilitation and then remains stable up to one year after discharge. There is a distinct paucity of studies in the medical literature describing long-term outcome of arm hand function.

The aim of the present study was to describe long-term outcome of AHSP in persons with CSCI. We studied the natural progression of AHSP using the VLT beyond one year after discharge. We also investigated association between the variables and the outcome. This may have implications for the duration of rehabilitation programmes for the tetraplegic upper limb and timing of reconstructive interventions.

\section{MATERIALS AND METHODS}

Participants

Data used for the present study was collected during a national research programme 'Physical strain, Work Capacity and Mechanisms of Restoration of Mobility in the Rehabilitation of Persons with Spinal Cord Injuries'. Eight rehabilitation centres in the Netherlands participated in the study. This was a prospective cohort study in which persons with an acute spinal cord injury admitted to these centres were followed from the onset of the active rehabilitation process to discharge from the centre and then follow-up measurements were carried out at 1 and 5 years after discharge.

\footnotetext{
${ }^{1}$ Rehabilitation Centre Het Roessingh, Enschede, The Netherlands; ${ }^{2}$ Reade Centre for Rehabilitation and Rheumatology, Amsterdam, The Netherlands; ${ }^{3} \mathrm{Center}$ for Human Movement Sciences, UMCG, University of Groningen, Groningen, The Netherlands; ${ }^{4}$ Adelante, Centre of Expertise in Rehabilitation, Hoensbroek, The Netherlands and ${ }^{5}$ Rudolf Magnus Institute of Neuroscience, University Medical Centre Utrecht and De Hoogstraat, Utrecht, The Netherlands Correspondence: Dr AC Franke, Rehabilitation Centre Het Roessingh, Postbus 310, 7500 AH, Enschede, The Netherlands. E-mail: C.Franke@zgt.nl

Received 6 December 2011; revised 19 June 2012; accepted 24 July 2012; published online 18 September 2012
} 
The criteria for the data to be included the present study were: persons with an acute CSCI (including thoracic 1-level), aged between 18 and 65 years and had no progressive disease. Data were excluded if persons had severe additional neurological, orthopaedic or rheumatologic diseases, or psychological problems that might interfere with the AHSP or if they did not understand the Dutch language well.

\section{Design}

Data were available of all persons who started their rehabilitation from the onset of their CSCI. For the study, five specific measurement moments were chosen to assess AHSP: $\mathrm{tl}$-at the start of active rehabilitation (it was termed as active rehabilitation because then the participants were able to sit consecutively for $3 \mathrm{~h}$ in a wheelchair, were free of their halo or corset and were able to start activity training); $\mathrm{t} 2$-at 3 months after the start; $\mathrm{t} 3$ - at discharge from the rehabilitation centre; $t 4$ - one year after discharge and $t 5-$ five years after discharge. The assessment protocol was approved by the medical ethics committees of all of the participating rehabilitation centres. Informed consent was given by all subjects.

\section{Measurements}

From the prospective cohort study the following data were available: age at the time of injury, gender and motor scores. Motor scores for each participant were determined on the basis of neurological assessment according to the 'International Standards for neurological and functional Classification of Spinal Cord Injury' (ISNCSCI) provided by the American Spinal Injury Association (ASIA). ${ }^{14}$

For the purpose of the present study a differentiation was made between motor complete (ASIA Impairment Scale (AIS) A and B) and motor incomplete lesions (AIS C and D). Furthermore a differentiation was also made between high CSCI (lesion level C3 to C6) and low CSCI (lesion level C7 to Th1).

The research version of the Van Lieshout test (VLT-SV, short version) was used to assess AHSP. This instrument was developed to objectively quantify the quality of AHSP in persons with a CSCI. ${ }^{12,13}$ According to the ICF model, it relates to the domain of (basic) activities. ${ }^{13}$ The VLT-SV includes 10 of the original 19 tasks. The possible ways of performing each task are described in six hierarchical levels, resulting in a score from 5 for the highest level of performance to 0 when the task cannot be performed at all. ${ }^{13}$ The total score can range from 0 to 50 . The best arm hand for each task was tested; as a result, in some persons a different arm hand was tested at different assessment moments and also within one assessment for different tasks.

The criterion validity, the inter-rater reliability as well as the internal consistency reliability of the VLT-SV is very good (Spearman's $r=0.87-0.90$, ICC $=0.98-0.99$, Cronbach's $\alpha=0.88-0.94$, respectively). The VLT-SV is sensitive to detect changes in AHSP during rehabilitation in persons with CSCI. ${ }^{12,13}$

Pain in the tested arm was evaluated by asking if subjects experienced pain in the upper extremity. The patients were asked at the time of the tests to indicate the presence or absence of pain in the tested arm.

Based on the available medical literature, following determinants were chosen out of the database for the purpose of analysing relationship between the AHSP and influencing factors: age, ${ }^{15}$ gender, ${ }^{16}$ motor score of upper extremity ${ }^{17}$ completeness of CSCI, ${ }^{17}$ and high vs low cervical lesion. ${ }^{18}$ Pain as an influencing factor on the outcome measure has rarely been studied. It was thought that it would be interesting to study the influence of pain on AHSP and hence it was also included.

\section{Data analysis}

At least three measurements needed to have been completed by each participant to be considered for analysis. Multilevel regression analysis $\left(\right.$ MLwiN) ${ }^{19}$ was used for statistical purposes. This method enables us to reach meaningful conclusions over the entire follow-up period inspite of missing data points including t1. As mentioned previously, the independent variables for this analysis were age at the time of injury, gender, motor complete or incomplete CSCI, total motor scores of the upper extremity, high (C3-C6) or low (C7-T1) CSCI level and pain in the tested arm.

The total scores of the VLT-SV were modelled over time using time periods as categorical variables (dummy), with the moment of discharge ( $\mathrm{t} 3$ ) as reference, that is, $\Delta \mathrm{t} 1 \mathrm{t} 3, \Delta \mathrm{t} 2 \mathrm{t} 3, \Delta \mathrm{t} 3 \mathrm{t} 4$ and $\Delta \mathrm{t} 3 \mathrm{t} 5$. The regression coefficient for a time period (for example, start to discharge; 3 months after start to discharge) describes the change of AHSP over that time period. An additional regression analysis was performed using the assessment 5 years after discharge (t5) as a reference, that is, $\Delta t 4 t 5$, to investigate the change in AHSP between 1 and 5 years after discharge. Statistical analysis programme SPSS for Windows (version 11.5) was used for this analysis.

Further, longitudinal relationships between the VLT-SV scores and the independent variables were investigated. All independent variables were measured during each assessment, except age at time of injury and gender (only collected at $\mathrm{t} 1$ ). The variables were added one by one to the basic model with the time dummies only. Independent variables with $P \leqslant 0.1$ were included in a subsequent multivariate model with a backward selection procedure, excluding non-significant determinants $(P>0.05)$ to create the final multivariate model.

\section{RESULTS}

The mean age of the participants was 38 years (s.d. 12.93) with a range from 18 to 64 years. Other characteristics of the participants are described in Table 1.

The mean VLT-SV scores over time are presented in Table 2. The mean score increased significantly during in-patient rehabilitation from 25 at t1 to 33 at $\mathrm{t} 3(\Delta \mathrm{t} 1 \mathrm{t} 3, P<0.005)$, but did not significantly change after in-patient rehabilitation $(\mathrm{t} 4=32$ and $\mathrm{t} 5=32)$

\section{Table 1 Patient characteristics}

\begin{tabular}{lcc}
\hline & $\mathrm{n}$ & $\%$ \\
\hline Total participants & 55 & 100 \\
Male & 40 & 73 \\
Female & 15 & 27 \\
C3-C6 & 49 & 89 \\
C7-Th1 & 6 & 11 \\
AIS AB & 38 & 69 \\
AIS CD & 17 & 31
\end{tabular}

\begin{tabular}{lcr} 
& $\begin{array}{c}\text { Number of } \\
\text { participants } n(\%)\end{array}$ & $\begin{array}{c}\text { Time since injury } \\
\text { Mean } \pm \text { s.d. in weeks }\end{array}$ \\
\hline t1 & $47(86)$ & $14 \pm 11.27$ \\
t2 & $45(82)$ & $31 \pm 12.10$ \\
t3 & $54(98)$ & $58 \pm 27.60$ \\
t4 & $42(76)$ & $117 \pm 30.22$ \\
t5 & $29(53)$ & $352 \pm 32.46$ \\
\hline
\end{tabular}

Values are $n$ or $\%$; mean \pm s.d. in weeks.

Table 2 The basic multilevel regression model for the total score of the VLT-SV

\begin{tabular}{|c|c|c|c|c|c|}
\hline \multicolumn{3}{|c|}{ VLT total score $t 3$ as reference } & \multicolumn{3}{|c|}{ VLT total score $t 5$ as reference } \\
\hline & $\beta$ (s.e.) & $\mathrm{P}$ & & $\beta$ (s.e.) & $\mathrm{P}$ \\
\hline Intercept & $32.618(2.850)$ & & Intercept & $31.655(3.256)$ & \\
\hline$\Delta \mathrm{t} 1 \mathrm{t} 3$ & $-7.941(2.839)$ & 0.005 & $\Delta \mathrm{t} 1 \mathrm{t} 5$ & $-6.977(3.270)$ & 0.033 \\
\hline$\Delta \mathrm{t} 2 \mathrm{t} 3$ & $-4.685(2.533)$ & 0.064 & $\Delta \mathrm{t} 2 \mathrm{t} 5$ & $-3.722(2.997)$ & 0.214 \\
\hline$\Delta \mathrm{t} 3 \mathrm{t} 4$ & $-0.591(2.582)$ & 0.819 & $\Delta \mathrm{t} 3 \mathrm{t} 5$ & 0.963 (2.899) & 0.740 \\
\hline$\Delta \mathrm{t} 3 \mathrm{t} 5$ & $-0.963(2.899)$ & 0.740 & $\Delta \mathrm{t} 4 \mathrm{t} 5$ & $0.372(3.037)$ & 0.903 \\
\hline
\end{tabular}

Abbreviation: VLT, Van Lieshout test.

Time dummies included only.

Values are the regression coefficient ( $\beta$ ) with the s.e. An increase in VLT score is indicated by a negative sign during in-patient rehabilitation, $\Delta \mathrm{t} 1 \mathrm{t} 3$ and $\Delta \mathrm{t} 2 \mathrm{t} 3$. A decrease in VLT score after in-patient rehabilitation, $\Delta \mathrm{t} 3 \mathrm{t} 4$ and $\Delta \mathrm{t} 3 \mathrm{t} 5$, is indicated by a negative sign. 
Table 3 Final multivariate regression model for the total score of the VLT-SV

\begin{tabular}{|c|c|c|}
\hline & \multicolumn{2}{|c|}{ VLT total score } \\
\hline & $\beta$ (s.e.) & $\mathrm{P}$ \\
\hline Intercept & $2.074(2.569)$ & \\
\hline$\Delta \mathrm{t} 1 \mathrm{t} 3$ & $-0.518(1.739)$ & 0.766 \\
\hline$\Delta \mathrm{t} 2 \mathrm{t} 3$ & $-1.239(1.350)$ & 0.359 \\
\hline$\Delta \mathrm{t} 3 \mathrm{t} 4$ & $0.474(1.379)$ & 0.731 \\
\hline$\Delta \mathrm{t} 3 \mathrm{t} 5$ & $10.258(2.816)$ & $<0.001$ \\
\hline Completeness & $-2.966(1.161)$ & $<0.001$ \\
\hline Motor score UE & $1.115(0.059)$ & $<0.001$ \\
\hline Pain tested arm & $-2.595(1.068)$ & 0.015 \\
\hline Lesion level & NS & \\
\hline Age & NS & \\
\hline Gender & NS & \\
\hline
\end{tabular}

Abbreviations: NS, independent variable was proven not significant in previous analyses and thus not entered into this model; UE, upper extremity; VLT, Van Lieshout test.

Values are the regression coefficient ( $\beta$ ) with the s.e. for the model after backward elimination. The regression coefficients represent the change in outcome associated with an increase of the independent variable of 1 unit.

Completeness: incomplete $=0$; complete $=1$.

Lesion level: $\mathrm{C} 3-\mathrm{C} 6=0 ; \mathrm{C} 7-\mathrm{T} 1=1$.

Pain in tested arm: not present $=0$; present $=1$.

( $\Delta \mathrm{t} 3 \mathrm{t} 5, P=0.740 ; \Delta \mathrm{t} 4 \mathrm{t} 5, P=0.903)$. Table 3 shows the results of the final multilevel regression model. After the backward selection procedure, three independent variables, namely, incomplete lesion $(P<0.001)$, high motor score upper extremity $(P<0.001)$ and no pain in the tested arm $(P=0.015)$ were found to be significantly related to the total score of the VLT-SV. The relationship was positive, that is, an incomplete lesion adds 3.0 extra points, no pain in the tested arm adds 2.6 points and every point of the MSUE adds 1.1 point to the total VLT-SV score. Lesion level, that is, high versus low, age and gender were not significantly related to the total score.

\section{DISCUSSION}

The aim of this study was to describe long-term results of AHSP assessed using the VLT-SV. The main findings were:

1. The score of the VLT-SV changed significantly during in-patient rehabilitation (in accordance with Spooren et al. ${ }^{8}$ ) but did not change significantly thereafter (up to 5 years after discharge).

2. Amongst the six independent variables tested, incomplete lesion, high MSUE and no pain in the tested arm were positively related to a higher score. Lesion level, that is, high versus low, age and gender did not have a significant relationship to the total score.

\section{Arm hand skilled performance}

For multilevel analysis t3 (end of in-patient rehabilitation) was chosen to enable comparison between the changes during the in-patient period and during the post-rehabilitation period. The in-patient period in the Netherlands is long compared with the other parts of the world. ${ }^{8}$ In this study, the mean time since injury at t 3 was about one year, $\mathrm{t} 3$ was unique for every patient representing reaching a plateau for motor performances since the time of injury. This is subscribed by the fact that the AHSP did not significantly improve after in-patient rehabilitation and remained stable in the years thereafter. This result coincides with the results of the earlier research. ${ }^{6,8}$ It is known that most spontaneous neurological recovery occurs in the first 3-9 months after injury. ${ }^{17,18}$ The period of in-patient rehabilitation in this study, during which improvement in AHSP occurred coincides with the period of most neurological recovery. As lengths of in-patient rehabilitation are or inclined to be much shorter in various parts of the world (including the Netherlands), clinicians have to be aware that plateau for the motor skills may not have been reached at the time of discharge and continuing training after discharge from in-patient rehabilitation and follow-up could be useful. ${ }^{8}$ Although Kirshblum and Fawcett ${ }^{17,18}$ have indicated that neurological recovery may continue until 18 months after injury, this study shows that AHSP does not improve after 1 year after injury. Usually consideration for reconstructive surgery is postponed until after the neurological recovery is complete, that is, for about 2 years after the injury. However, results of this study show that there is no further improvement in AHSP after 1 year after the injury and hence reconstructive surgery for improvement of arm hand function could be considered and performed after 1 year after the date of injury.

\section{Independent variables}

We studied six independent variables. Three variables, namely, incomplete lesion, high motor score upper extremity and no pain in tested arm, turned out to be statistically relevant in improving the VLT score. Several studies also conclude that an incomplete lesion and a high motor score of the upper extremity have a better functional prognosis. ${ }^{8,17,18}$ Pain is infrequently investigated. It could be that pain in the tested arm leads to diminished functioning due to avoidance or diminished motivation. We did take pain into consideration; however, it was not studied in detail. Hence, besides indicating the influence of pain in AHSP we cannot make any further comments. In future, the relationship between pain and AHSP needs to be studied in greater detail.

Surprisingly, the lesion level was not significantly related to the outcome. Looking back, there were only six subjects with low-level CSCI and out of these, three were determined by sensory level. This number is too low to reach statistical significance.

\section{Methodological considerations}

There are some methodological limitations to this study. First, only 29 of the 55 participants performed the VLT-SV at t5. Loss-to-follow-up and missing values is a common problem encountered in the longitudinal studies, this was true for this study as well. Use of multilevel regression analysis was an attempt to minimise this problem.

Second, there is a ceiling effect in test performance. In this study, subjects with spinal cord lesions from C3 to T1 were included. It is well known that in subjects with a high initial MSUE, the incidence of arm hand function problems is lower.

Last, the best arm hand was tested during measurement chosen by the patient. However, 24 subjects used the same hand throughout all the tests. Sixteen subjects out of the 25 subjects who completed both $t 4$ and $t 5$ used the same hand on both occasions. One might argue that the results of measuring the same arm hand are more accurate. However, the VLT is placed at the activity level according to the ICF model. ${ }^{13,20}$ This implies that it measures the functional abilities of the subject using his or her best arm hand in performing daily skills. This best arm could be different at different measurement moments or different for different tasks. It was thought to be important to obtain the best functional result instead of measuring the improvement of AHSP in one specific arm hand. 


\section{CONCLUSION}

This is the first study evaluating long-term outcome of AHSP at activity level in patients with CSCI.

One can conclude from this study that the AHSP measured by the VLT-SV improves significantly during in-patient rehabilitation, the average duration of this period was 58 weeks, after that period AHSP did not change significantly. Continuation of rehabilitation programme to improve AHSP may not be necessary after 1 year and arm hand reconstructive surgery could be considered then on.

Significant relationship exists between VLT-SV scores and completeness of lesion, MSUE and pain in the tested arm. Lesion level, that is, high versus low, age and gender do not have a significant relationship to the total score.

In previous studies in the literature, pain has not received much attention. We have shown that there exists a relationship between AHSP and the presence of pain. Future studies should examine this relationship in greater detail as well as whether effective pain management could improve performance.

\section{DATA ARCHIVING}

There were no data to deposit.

\section{CONFLICT OF INTEREST}

The authors declare no conflict of interest.

\section{ACKNOWLEDGEMENTS}

The present study is part of the research programme 'physical strain, work capacity and mechanisms of restoration of mobility in rehabilitation of individuals with a spinal cord injury'. We thank the eight participating rehabilitation centres for their effort and data.

1 Wyndaele M, Wyndaele J-J. Incidence, prevalence and epidemiology of spinal cord injury: what learns a worldwide literature survey? Spinal cord 2006; 44: 523-529.

2 Anderson KD. Targeting recovery: priorities of the spinal cord-injured population J Neurotrauma 2004; 21: 1371-1383.
3 Spooren AIF, Janssen-Potten YIM, Kerckhofs E, Seelen HAM. Outcome of moto training programmes on arm and hand functioning in patients with cervical spinal cord injury according to different levels of the ICF: a systematic review. J Rehabil Med 2009; 41: 497-505.

4 Snoek GJ, IJzerman MJ, Hermens HJ, Maxwell D, Biering-Sorensen F. Survey of the needs of patients with spinal cord injury: impact and priority for improvement in hand function in tetraplegics. Spinal Cord 2004; 42: 526-532.

5 Hanson RW, Franklin MR. Sexual loss in relation to other functional losses for spina cord injured males. Arch Phys Med Rehabil 1976; 57: 291-293.

6 Dzidic I, Moslavac S. Functional skills after the rehabilitation of spinal cord injury patients: observation period of 3 years. Spinal Cord 1997; 35: 620-623.

7 Yarkony GM, Roth EJ, Heinemann AW, Lovell L, Wu YC. Functional skills after spinal cord injury rehabilitation: three year longitudinal follow-up. Arch Phys Med Rehab 1988; 69: 111-114.

8 Spooren A, Janssen-Potten YJ, Snoek GJ, Ijzerman MJ, Kerckhofs E, Seelen HA Rehabilitation outcome of upper extremity skilled performance in persons with cervical spinal cord injuries. J Rehabil Med 2008; 40: 637-644.

9 Yavuz N, Tezyurek M, Akyuz M. A comparison of two functional tests in quadriplegia: the quadriplegia index of function and the functional independence measure. Spinal Cord 1998; 36: 832-837.

10 Harvey LA, Batty J, Jones R, Crosbie J. Hand function of C6 and C7 tetraplegics $1 \pm 16$ years following injury. Spinal Cord 2001; 39: 37-43.

11 World Health Organization. ICF: International Classification of Functioning, Disability and Health. Geneva, World Health Organisation, 2001.

12 Spooren AIF, Janssen-Potten YIM, Post MWM, Kerckhofs E, Nene AV, Seelen HAM Measuring change in arm hand skilled performance in persons with a cervical spinal cord injury: responsiveness of the Van Lieshout Test. Spinal Cord 2006; 44: 772-779.

13 Post MWM, Van Lieshout G, Seelen HAM, Snoek GJ, IJzerman MJ, Pons C. Measurement properties of the short version of the Van Lieshout test for arm hand functioning of persons with tetraplegia after spinal cord injury. Spinal Cord 2006; 44 763-711.

14 American Spinal Injury Association/International Medical Society of Paraplegia. International Standards for Neurological and Functional Classification of Spinal Cord Injury Patients. American Spinal Injury Association: Chicago, 2000.

15 Marino RJ, Stineman MG. Functional assessment in spinal cord injury. Top Spinal Cord Inj Rehabil 1996; 1: 32-45.

16 Sipski ML, Jackson AB, Gómez-Marín O, Estores I, Stein A. Effects of gender on neurologic and functional recovery after spinal cord injury. Arch Phys Med Rehabil 2004; 85: 1826-1836

17 Fawcett JW, Curt A, Steeves JD, Coleman WP, Tuszynski MH, Lammertse D et al. Guidelines for the conduct of clinical trials for spinal cord injury as developed by the ICCP panel: spontaneous recovery after spinal cord injury and statistical power needed for therapeutic clinical trials. Spinal Cord 2007; 45: 190-205.

18 Kirshblum SC, O'Connor KC. Predicting neurologic recovery in traumatic cervical spina cord injury. Arch Phys Med Rehabil 1998; 79: 1456-1466.

19 Twisk J. Applied Longitudinal Data Analysis for Epidemiology. A Practical Guide. Cambridge Univ Pr: Cambridge, 2003.

20 Ditunno JF. Outcome measures: evolution in clinical trials of neurological/functional recovery in spinal cord injury. Spinal Cord 2010; 48: 674-684. 